\title{
EXPERIMENTAL STUDY OF THE PYGMY DIPOLE RESONANCE IN THE ${ }^{68} \mathrm{Ni}$ NUCLEUS*
}

\author{
N.S. Martorana ${ }^{a, b}$, G. Cardella ${ }^{\mathrm{c}}$, E.G. Lanza $^{\mathrm{c}}$, L. Acosta $^{\mathrm{d}}$ \\ M.V. Andrés ${ }^{e}$, L. Auditore ${ }^{f}, F$. Catara ${ }^{c}$, E. De Filippo ${ }^{c}$
}

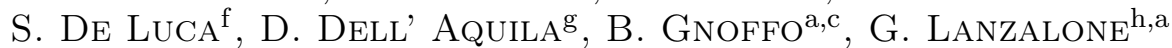

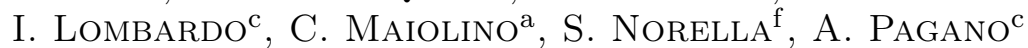
E.V. PAGANO ${ }^{\mathrm{a}}$, M. PAPA ${ }^{\mathrm{c}}$, S. PirRONE ${ }^{\mathrm{c}}$, G. Politi ${ }^{\mathrm{a}, \mathrm{c}}$

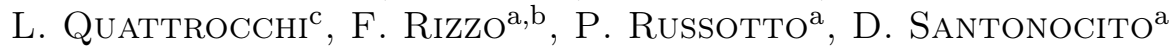

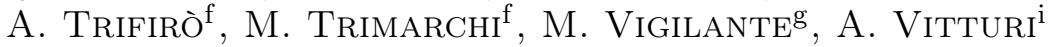

${ }^{a}$ LNS-INFN, Catania, Italy

${ }^{\text {b}}$ Dipartimento di Fisica e Astronomia, Università degli studi di Catania Catania, Italy

${ }^{\mathrm{C}}$ INFN — Sezione Catania, Catania, Italy

${ }^{\mathrm{d}}$ Instituto de Física, Universidad Nacional Autónoma de México

Mexico City, Mexico

e Departamento de FAMN, Universidad de Sevilla, Sevilla, Spain

${ }^{\mathrm{f}}$ Dipartimento MIFT, Messina, Italy

'INFN — Sezione di Napoli, Napoli, Italy

and

Dipartimento di Fisica Università di Napoli Federico II, Napoli, Italy

${ }^{h}$ Facoltà di Ingegneria e Architettura, Università Kore, Enna, Italy

${ }^{i}$ Dipartimento di Fisica, Università G. Galilei, Padova, Italy

and

INFN — Sezione di Padova, Padova, Italy

(Received January 3, 2018)

It is known that the low-energy E1 strength, known as the Pygmy Dipole Resonance (PDR), can be excited by both isovector and isoscalar probes. In this work, we report on the results of an experiment, carried out at the LNS-INFN in Catania, with the aim to study the Pygmy Dipole Resonance induced in the ${ }^{68} \mathrm{Ni}$ nucleus through the interaction with a ${ }^{12} \mathrm{C}$ isoscalar target. The CHIMERA multidetector and the FARCOS array were used to detect, respectively, $\gamma$ rays and charged particles. Details on the experimental set-up, data analysis and results are reported.

DOI:10.5506/APhysPolB.49.475

* Presented at the XXXV Mazurian Lakes Conference on Physics, Piaski, Poland, September 3-9, 2017. 


\section{Introduction}

The Pygmy Dipole Resonance (PDR) is an excitation mode that is related to the neutron excess in nuclei; several microscopic models predict that it is present in all stable nuclei with neutron excess and, in particular, in unstable nuclei [1-4]. The investigation of the PDR is currently a topic of interest due to its relation to the Equation of State of nuclear matter (EoS) $[5,6]$ and to the $\mathrm{r}$ process, responsible for the nucleosynthesis of heavy elements [7-9]. Microscopic calculations show that in this mode, the neutrons and protons transition densities are in phase inside the nucleus, while at the surface, just the neutron part survives; this property may be considered to be the theoretical definition of the PDR [1, 10-15]. Another important feature, evidenced by the calculations, is that the strengths of the isoscalar and isovector parts of the transition density at the surface are of the same order of magnitude. This means that it is possible to induce the excitation of the Pygmy mode by using both isoscalar and isovector probes [15].

Until now, several experiments on stable nuclei have been performed by using both isoscalar and isovector probes $[1,2,5]$. The presence of the PDR in unstable nuclei was investigated using the relativistic Coulomb reactions, aiming at exciting the PDR in several isotopes [16-21]. In this work, we report on the results of the study of the PDR induced in the ${ }^{68} \mathrm{Ni}$ isotope by means of an isoscalar probe, namely a ${ }^{12} \mathrm{C}$ target. The experiment was performed at the LNS-INFN in Catania and the data analysis concentrated on the $\gamma$-decay channel above the neutron-emission threshold.

\section{Experimental setup}

Unstable ${ }^{68} \mathrm{Ni}$ nuclei were produced using the In-Flight Fragmentation method at the FRIBs (in-Flight Radioactive Ion Beams) facility of the LNSINFN [22]. We used a primary beam of ${ }^{70} \mathrm{Zn}$ accelerated by the Superconducting Cyclotron (CS) to the energy of $40 \mathrm{~A} \mathrm{MeV}$, impinging on a target of ${ }^{9} \mathrm{Be}, 250 \mu \mathrm{m}$ thick. A fundamental component of this facility is the standard tagging system of the NEWCHIM-CHIMERA Collaboration [23], which allows measuring event-by-event the isotopic composition of the exotic beam. The tagging system is composed of a Micro-Channel Plate detector (MCP), with a surface of $43 \mathrm{~mm} \times 63 \mathrm{~mm}$, and a $2 \mu \mathrm{m}$ Mylar foil, and of a DoubleSided Silicon Strip Detector (DSSSD), $32 \mathrm{~mm} \times 32 \mathrm{~mm}, 156 \mu \mathrm{m}$ thick. The identification of nuclei forming the exotic beam is obtained by using the $\Delta E-$ ToF method. The start of the Time of Flight (ToF) was given by the MCP, located at a distance of $12.9 \mathrm{~m}$ from the DSSSD. The stop of the ToF and the $\Delta E$ were provided by the strips of the DSSSD, mounted about $2 \mathrm{~m}$ before the ${ }^{12} \mathrm{C}$ reaction target. The tagging system was also used to obtain the position information, important for the trajectory reconstruction of the 
beam. In order to determine the beam trajectory more precisely, we used a Parallel Plate Avalanche Counter (PPAC) with a spatial resolution of $1 \mathrm{~mm}$, mounted $80 \mathrm{~cm}$ from the $75 \mu \mathrm{m}{ }^{12} \mathrm{C}$ target.

The PDR can decay via both neutron and $\gamma$-ray emission; in this work, we focus on the study of the $\gamma$-ray decay channel. To detect the $\gamma$ rays, we used the CHIMERA (Charged Heavy Ion Mass and Energy Resolving Array) multidetector [24], whereas to detect the ${ }^{68} \mathrm{Ni}$ and other heavy ions, produced in the reaction with ${ }^{12} \mathrm{C}$, we used the FARCOS (Femtoscope ARray For COrrelations and Spectroscopy) array [25, 26].

The CHIMERA multidetector (Fig. 1, left) includes 1192 telescopes, each of them consisting of a silicon detector followed by a $\mathrm{CsI}(\mathrm{Tl})$. The CHIMERA multidetector is divided in two main parts: the forward part, consisting of 688 telescopes and covering the $\theta$ angles from $1^{\circ}$ to $30^{\circ}$, and the backward part, with 504 telescopes covering the angles from $30^{\circ}$ to $176^{\circ}$. The backward part telescopes form a sphere around the target, with a radius of $40 \mathrm{~cm}$. In order to detect the $\gamma$ rays, we used the sphere of CHIMERA, as shown in Ref. [27].
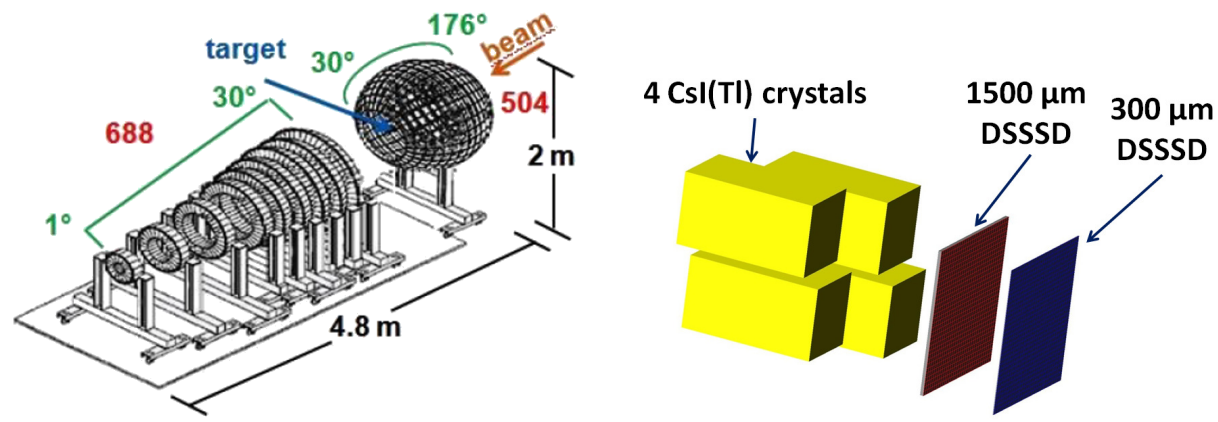

Fig. 1. Left: Scheme of the CHIMERA multidetector. Right: Scheme of a FARCOS array telescope.

The FARCOS array consists of four telescopes, each of them having a configuration shown in Fig. 1, right, with two Double-Sided Silicon Strip Detectors $(32 \times 32$ strips) of a thickness of $300 \mu \mathrm{m}$ (first stage) and $1500 \mu \mathrm{m}$ (second stage). These telescopes are followed by $4 \mathrm{CsI}(\mathrm{Tl}), 6 \mathrm{~cm}$ thick. During the experiment, FARCOS was placed just after the sphere of CHIMERA and covered $\theta$ angles from $2^{\circ}$ to $7^{\circ}$, with a $70 \%$ coverage of the azimuthal angle. 


\section{Data analysis}

The first part of the data analysis was the calibration of the tagging system. In Fig. 2 (a), the $\Delta E-$ ToF plot obtained with the tagging system is reported. As can be seen, the exotic beam is composed of different elements with ${ }^{68} \mathrm{Ni}$ being the most abundant, amounting to $20 \%$ of the total beam intensity. The beam energy after the tagging detector is around $28 \mathrm{~A} \mathrm{MeV}$, which makes the ion identification difficult due to the presence of various charge states produced in the reactions with ${ }^{9} \mathrm{Be}$. However, thanks to the configuration of the transport beam line with magnets after the MCP, most of these charge states are rejected. After the interaction with the ${ }^{12} \mathrm{C}$ target, ${ }^{68} \mathrm{Ni}$ and other heavy ions were detected using the FARCOS array. At this energy, most ions deposit the total energy in the second stage of the FARCOS array without reaching the $\mathrm{CsI}(\mathrm{Tl})$. In Fig. $2(\mathrm{~b})$, a $\Delta E-E$ plot obtained with this array is shown. The $\Delta E-E$ plot is reported in channels, as due to the energy spread of the fragmentation beam $(2 \%)$ and the angular spread, it is difficult to extract the absolute energy calibration. However, a good identification has been obtained, partly thanks to the cleaning effect of the fragment separator. In the upper panel of Fig. 2 (b), a $\Delta E-E$ plot is shown corresponding to the events where the ${ }^{68} \mathrm{Ni}^{28+}$ beam has been detected with the tagging system.
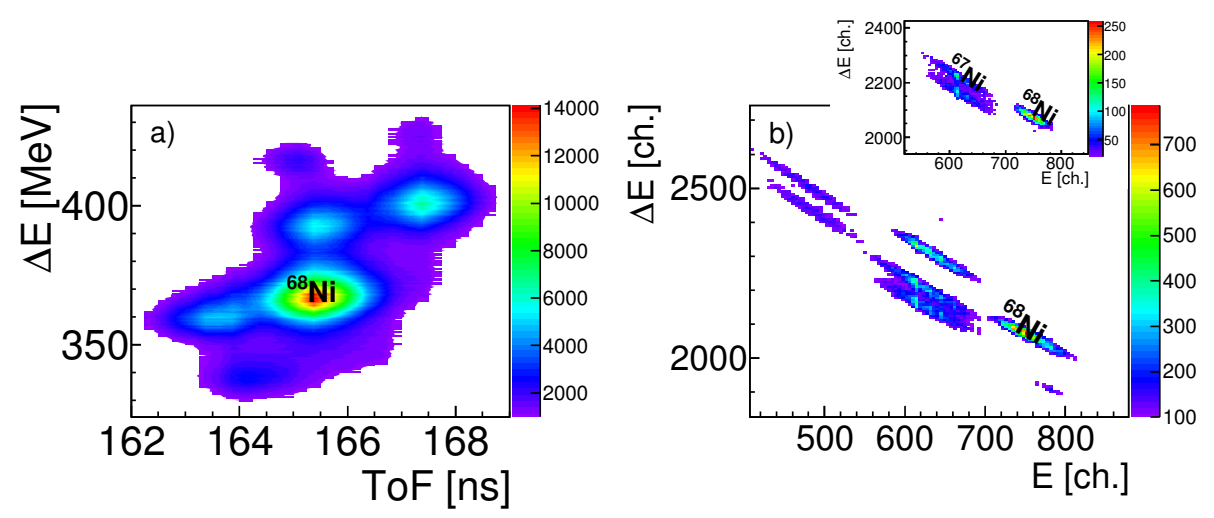

Fig. 2. (a) $\Delta E$-ToF plot obtained with the tagging system placed at the entrance of the CHIMERA chamber. (b) $\Delta E-E$ plot of reaction products obtained with the FARCOS array. In the upper panel, the $\Delta E-E$ plot in coincidence with the ${ }^{68} \mathrm{Ni}^{28+}$ incoming beam is reported.

As mentioned above, to detect the $\gamma$ rays the sphere of the CHIMERA multidetector was used, in particular the $\mathrm{CsI}(\mathrm{Tl})$ scintillators forming its second stage. The calibration of $\mathrm{CsI}(\mathrm{Tl})$ was performed using a proton beam of $24 \mathrm{MeV}$, delivered by the TANDEM accelerator of the LNS-INFN, and 
impinging on ${ }^{12} \mathrm{C},{ }^{197} \mathrm{Au}$ and $\mathrm{CH}_{2}$ targets. We used the elastic and inelastic reactions of the proton beam as well as a pulser to calibrate the detectors in proton-equivalent energy and also to verify the linearity of the response function. In Fig. 3 (a), we report the $Q$-value spectrum for the $p+{ }^{12} \mathrm{C}$ reaction. The protons are detected in all telescopes of the CHIMERA multidetector sphere. In Fig. $3(\mathrm{~b})$, we report the $\gamma$-ray energy spectra in coincidence with the two different windows of $Q$-value. These two peaks correspond to the elastic channel (grey/green spectrum) and the inelastic channel (light grey/yellow) [28]. The latter is associated to the $4.44 \mathrm{MeV}$ level produced in the ${ }^{12} \mathrm{C}$ decay. The background under this peak was evaluated by means of the coincidence with the elastic channel (green spectrum). The subtraction of the two spectra is shown in Fig. 3 (c).
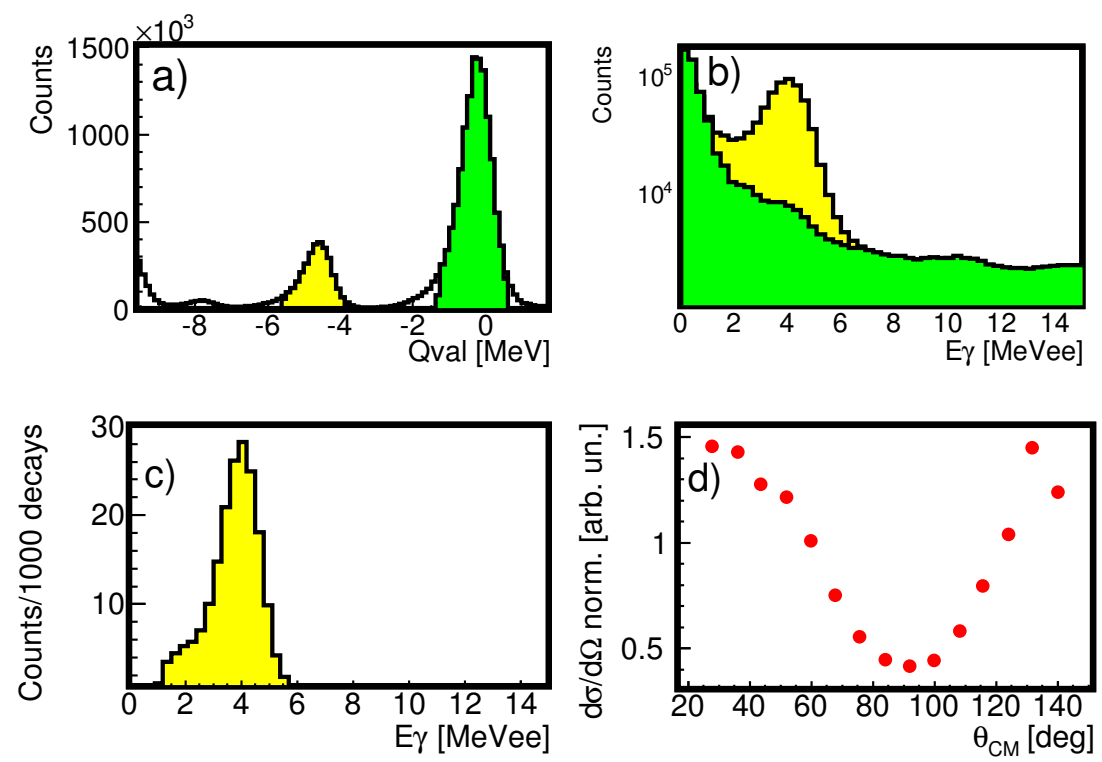

Fig. 3. (a) $Q$-value spectrum for the $p+{ }^{12} \mathrm{C}$ reaction. The protons are detected in all telescopes of the CHIMERA multidetector sphere. (b) Energy spectra of $\gamma$ rays detected with the $\mathrm{CsI}(\mathrm{Tl})$ of CHIMERA, in coincidence with the inelastic (light grey/yellow peak) and the elastic channel (grey/green peak) of the $p+{ }^{12} \mathrm{C}$ reaction. (c) Energy spectra of $\gamma$ rays detected with the CHIMERA CsI(Tl), resulting from the subtraction of the two $\gamma$-ray spectra shown in panel (b). (d) angular distribution for decay of the $4.44 \mathrm{MeV}$ level [27].

This calibration was also used to check if $\gamma$-ray angular distributions could be extracted. In Fig. 3 (d), the angular distribution for the $4.44 \mathrm{MeV}$ level is reported. The observed trend is in agreement with a typical distribution for a $2^{+} \rightarrow 0^{+}$transition, see Ref. [27] for details. 
Moreover, we want to highlight that we do not observe $\gamma$ rays in the region around $10 \mathrm{MeV}$, where the PDR is expected, due to the decay of ${ }^{12} \mathrm{C}$. This is due to the very low $\gamma$-decay branching ratio of high-energy levels in ${ }^{12} \mathrm{C}$. We also underline that the $\gamma$ rays could not be discriminated from particles for the TANDEM experiments, resulting in the large background shown in Fig. 3(b), while for fragmentation beams the fast-slow identification technique [29] was possible by using the MCP signal as the starting time for the slow gate.

\section{Results}

Looking at the upper panel of Fig. 2 (b), we note that most of the events correspond to the production of ${ }^{66,67} \mathrm{Ni}$, hence to the emission of one or two neutrons, while in a smaller number of events, the beam particles are detected. The elastic channel is not observed due to the coverage angles of the FARCOS array; the grazing angle is in fact around $0.7^{\circ}$. With the aim to study the $\gamma$-ray decay of the PDR, we collected the $\gamma$-ray energy spectra in coincidence with the neutron-emission channels and with the ${ }^{68} \mathrm{Ni}$ quasi-elastic channel, as shown in Fig. 4 (a). The $\gamma$-ray energy spectrum in coincidence with the ${ }^{68} \mathrm{Ni}$ reaction channel is denoted with black dots, whereas that in coincidence with ${ }^{66,67} \mathrm{Ni}$ is reported as red squares. The spectra are Doppler-shift corrected with respect to the velocity of the projectile. According to the literature [3, 18], the PDR should be observed in the ${ }^{68} \mathrm{Ni}$ reaction channel. Indeed, in the black spectrum an enhancement around $10 \mathrm{MeV}$ is visible. Such enhancement is not observed in the ${ }^{66,67} \mathrm{Ni}$ reaction channel, where the neutron emission decreases the excitation energy of the system, inhibiting the emission of high-energy $\gamma$ rays. The spectra have a similar shape in the region from 2 to $6 \mathrm{MeV}$, which means that the
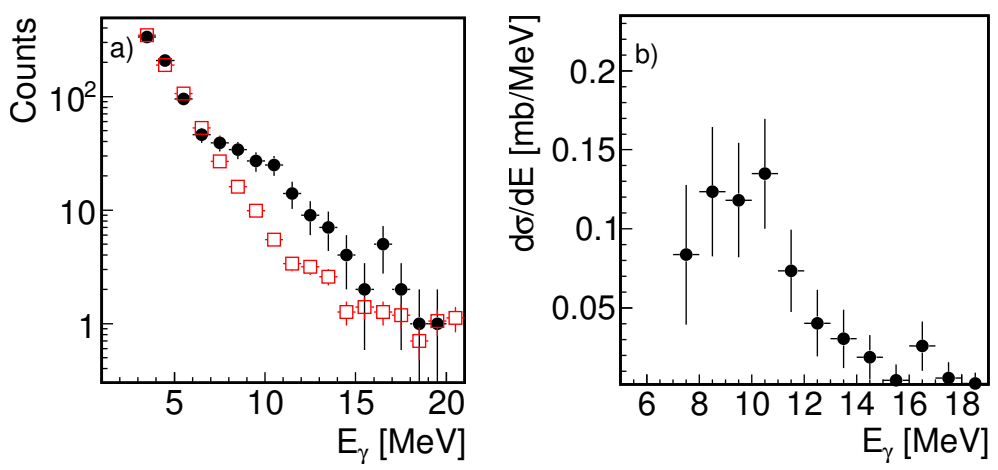

Fig. 4. (a) $\gamma$-ray energy spectra, Doppler-shift corrected, in coincidence with the ${ }^{68} \mathrm{Ni}$ (black dots) and ${ }^{66,67} \mathrm{Ni}$ (red squares) reaction channels. (b) Yield of the PDR obtained from the subtraction of the spectra in figure (a). 
sources of the $\gamma$ decay have similar characteristics. The red spectrum has instead an exponential behavior at high energy. It can be used to show a preliminary location of the PDR. In the high-energy region, also the Giant Dipole Resonance (GDR) seems to be excited in the ${ }^{68} \mathrm{Ni}$ reaction channel, but due to the low cross section and low $\gamma$-ray detection efficiency in this energy range we cannot extract the information about the GDR decay.

The cross section for the PDR obtained from the subtraction of the two spectra is shown, in linear scale, in Fig. 4(b). To calculate it, the total beam intensity, target thickness, $\gamma$-ray detection efficiency and ${ }^{68} \mathrm{Ni}$ detection efficiency have been taken into account.

\section{Conclusion}

We have observed for the first time, the excitation of the PDR in the unstable nucleus ${ }^{68} \mathrm{Ni}$ using an isoscalar probe. Thanks to the tagging system, we determined the isotopic composition of the exotic beam and evaluated the total intensity of the ${ }^{68} \mathrm{Ni}$ beam. The $\gamma$ rays were detected with the CHIMERA multidetector, with a possibility to extract their angular distribution. As expected, we observed the $\gamma$ decay of the PDR in the region around $10 \mathrm{MeV}$ in the ${ }^{68} \mathrm{Ni}$ reaction channel. The comparison with the ${ }^{66,67} \mathrm{Ni}$ reaction channel allows for a preliminary evaluation of the PDR yield and the cross section for the $\gamma$ decay. In order to better evaluate the background in the PDR region, we are performing a statistical calculation with the CASCADE code [30]. This analysis, as well as the extraction of the angular distribution of the PDR, very important to verify the E1 character of the transition, is still on-going and will be a subject of a forthcoming paper.

\section{REFERENCES}

[1] N. Paar et al., Rep. Prog. Phys. 70, 691 (2007) and references therein.

[2] D. Savran et al., Prog. Part. Nucl. Phys. 70, 210 (2013).

[3] A. Bracco et al., Eur. Phys. J. A 51, 99 (2015).

[4] F. Catara, E.G. Lanza, M.A. Nagarajan, A. Vitturi, Nucl. Phys. A 614, 86 (1997); 624, 449 (1997).

[5] A. Klimkiewicz et al., Phys. Rev. C 76, 051603(R) (2007).

[6] J. Piekarewicz et al., Phys. Rev. C 73, 044325 (2006).

[7] S. Goriely, E. Khan, Nucl. Phys. A 706, 217 (2002).

[8] N. Tsoneva et al., Phys. Rev. C 91, 044318 (2015).

[9] E. Litvinova et al., Nucl. Phys. A 823, 26 (2009).

[10] D. Vretenar et al., Nucl. Phys. A 692, 496 (2001). 
[11] N. Paar et al., Phys. Lett. B 606, 288 (2005).

[12] E. Litvinova, Phys. Lett. B 647, 111 (2007).

[13] N. Tsoneva, H. Lenske, Phys. Rev. C 77, 024321 (2008).

[14] E.G. Lanza et al., Phys. Rev. C 79, 054615 (2009).

[15] E.G. Lanza et al., Phys. Rev. C 84, 064602 (2011).

[16] A. Leistenschneider et al., Phys. Rev. Lett. 86, 5442 (2001).

[17] E. Tryggestad et al., Phys. Lett. B 541, 52 (2002).

[18] P. Adrich et al., Phys. Rev. Lett. 95, 132501 (2005).

[19] J. Gibelin et al., Phys. Rev. Lett. 101, 212503 (2008).

[20] O. Wieland et al., Phys. Rev. Lett. 102, 092502 (2009).

[21] D.M. Rossi et al., Phys. Rev. Lett. 111, 242503 (2013).

[22] https://www.lns.infn.it/it/acceleratori/fribs-lns.html

[23] I. Lombardo et al., Nucl. Phys. B Proc. Suppl. 215, 272 (2011).

[24] A. Pagano et al., Nucl. Phys. A 734, 504 (2004).

[25] E.V. Pagano et al., Eur. Phys. J. Web. Conf. 117, 10008 (2016).

[26] L. Acosta et al., J. Phys.: Conf. Ser. 730, 012001 (2016).

[27] G. Cardella et al., Nucl. Instrum. Methods Phys. Res. A 799, 64 (2015).

[28] G. Cardella et al., Nuovo Cim. C 39, 407 (2016).

[29] M. Alderighi et al., Nucl. Instrum. Methods Phys. Res. A 489, 257 (2002).

[30] F. Pühlhofer, Nucl. Phys. A 280, 267 (1977). 